\title{
Establishment and analysis of a mouse model that regulates sex- related differences in liver drug metabolism
}

\author{
Hiromi Chikada ${ }^{1,2} \cdot$ Kinuyo Ida $^{1} \cdot$ Emi Ando $^{1} \cdot$ Yutaka Inagaki $^{2} \cdot$ Akemi Sakamoto $^{3} \cdot$ Akihide Kamiya $^{1}$
}

Received: 21 June 2017 / Revised: 3 April 2018 / Accepted: 25 April 2018 / Published online: 3 July 2018

(c) United States \& Canadian Academy of Pathology 2018

\begin{abstract}
The adult liver performs many metabolic functions for maintaining homeostasis. There are several sex differences in liver function and disease pathogenesis. One important function of the liver is drug metabolism, where cytochrome p450s (CYPs) in hepatocytes are the main enzymes involved. The toxicity of various drugs and chemicals differs with sex due to differences in hepatocytic CYP expression. However, the molecular mechanism regulating sex-related differences in drug metabolism remains unknown. In this study, we identified transcriptional regulator B-cell lymphoma 6 (Bcl6) as an important factor in sex-biased differential CYP expression. Microarray analysis of livers derived from liver-specific Bcl6-knockout mice showed that Bcl6 is required for sex-biased CYP expression patterns in the liver. Additionally, quantitative PCR analysis revealed that hepatocytic expression of male-biased genes, such as Cyp2d9, Cyp2u1, Cyp4a12a/12b, and Cyp7b1, in liver-specific Bc16-knockout male mice significantly decreased to levels similar to those observed in wild-type female mice. Conversely, hepatocytic expression of female-biased genes, such as Cyp2a4/2a5, Cyp2b9, Cyp3a41, and Cyp17a1, significantly increased in liver-specific Bcl6knockout male mice. Deletion of Bcl6 caused female-like expression of CYPs in male livers. These results suggest that Bcl6 is a key regulator of sex-related differential regulation of drug metabolism. Moreover, serum sex hormone levels and fertility did not change in liver-specific, Bcl6-knockout mice. Hepatocytic Bcl6 regulates sex-related differential CYP expression in the liver without changing the sex of the whole body. Thus, this mouse model is useful for analyzing liver-specific sex-dependent regulation of drug metabolism and pathogenesis.
\end{abstract}

\section{Introduction}

Sexually dimorphic characteristics are derived from sexbased gene expression. In addition to reproductive organs, gene expression profiles in several tissues, including the liver, are different between males and females [1]. The liver

Electronic supplementary material The online version of this article (https://doi.org/10.1038/s41374-018-0088-6) contains supplementary material, which is available to authorized users.

Akihide Kamiya

kamiyaa@ tokai-u.jp

1 Department of Molecular Life Sciences, Tokai University School of Medicine, 143 Shimokasuya, Isehara, Kanagawa 259-1193, Japan

2 Department of Regenerative Medicine, Center for Matrix Biology and Medicine, Tokai University School of Medicine, 143 Shimokasuya, Isehara, Kanagawa 259-1193, Japan

3 Biomedical Research Center, Chiba University, 1-8-1 Inohana, Chuo-ku, Chiba-city, Chiba 260-8670, Japan plays an important role in maintaining homeostasis through processes such as nutrient metabolism and storage, drug detoxification, and plasma protein synthesis. One of the most important functions of the liver is drug metabolism. Drugs are metabolized through a two-step chemical reaction and are then excreted through the liver or kidney. The drugs are first hydroxylated by cytochrome P450s (CYPs) to increase drug solubility. Next, endogenous compounds such as sulfuric acid and glutathione are conjugated to the drugs to allow for more efficient drug excretion. CYPs are hemecontaining, membrane-bound enzymes that play important roles in the metabolism of lipophilic compounds. These proteins belong to large super families, such as CYP1, CYP2, and CYP3, where each superfamily in turn consists of subfamilies, such as CYP1A and CYP1B. Different species express varying amounts of putative functional CYP genes; for example, there are almost 50 CYP genes in humans and 100 in mice [2]. Drug pharmacokinetics and pharmacodynamics are known to differ with sex because CYPs are differentially expressed between the two sexes in both rodents and humans [3]. For instance, Cyp2d9 is 
mainly expressed in male mice [4], whereas Cyp3a41 is mainly expressed in females [5]. In humans, CYP3A activity is higher in females than in males [6], and as methylprednisolone is mainly metabolized by CYP3A, it is eliminated faster in females [7]. Drug responses and pathological conditions of the liver are sometimes regulated in a sex-differential manner. Therefore, both male and female subjects are necessary for clinical trials during drug discovery. However, increasing the number of female subjects during the early stages of clinical trials is difficult, because pregnant or fertile women cannot participate. It is thus advantageous to develop an animal model for evaluating sex-biased drug metabolism in drug discovery.

CYP expression is regulated in a sex-differential manner through plasma growth hormone $(\mathrm{GH})$ profiles [8], which are sexually dimorphic in many species. In males, GH is secreted from the somatotrophs of the anterior pituitary gland in a pulsatile manner, allowing for $\mathrm{GH}$-free or $\mathrm{GH}$ low intervals between peak plasma GH levels. In contrast, $\mathrm{GH}$ secretion is more frequent and continuous in female plasma [8]. This difference in plasma GH profiles between males and females is important for sex-differential gene expression. When continuous GH was infused in male mice using an osmotic pump, downregulation of male-biased genes and upregulation of female-biased genes was observed [9]. GH signals activate STAT5b via phosphorylation, which then translocates into the nucleus to regulate the expression of its target genes [10]. STAT5b is one of the key regulators of sex-differential gene expression as demonstrated in STAT5b-knockout mice [11, 12]. However, the molecular mechanism regulating sex-biased hepatocytic CYP expression remains unknown. Determining the key factors for this mechanism may be useful for drug discovery and analysis of sex-related differences in liver pathogenesis.

B-cell lymphoma 6 (Bcl6) was first identified from the translocation breakpoint in lymphomas [13]. Bcl6 is a $\mathrm{C} 2 \mathrm{H} 2$-type zinc finger transcriptional repressor that plays an important role in the formation of germinal center B cells $[14,15]$. Moreover, Bcl6 binds the consensus DNA sequence of STAT5b in the liver [16, 17]. These results suggest that Bcl6 may be involved in the sex-related differences in liver functions. Bcl6-knockout mice cannot form germinal center B cells and thus develop myocarditis [18, 19]. In addition, expression of Bcl6 in the liver and adipose tissues is required for lipid metabolism, as Bcl6 deficiency causes a marked decrease in weight and adipose tissue mass [20]. Therefore, most Bcl6-knockout mice die within 5 weeks of birth. As a result, analysis of Bcl6 function in the adult liver is difficult. In this study, we generated liverspecific Bcl6-knockout mice to analyze the relationship between Bcl6 and sex-related differences in the liver focusing on sex-differential drug metabolic genes. The sex- biased expression of CYPs was abolished in liver-specific Bcl6-knockout mice. Moreover, sex steroid hormone levels in serum and the reproductive abilities of mice did not change after deletion of Bcl6 in hepatocytes. These results imply that Bcl6 controls sex-driven differences in the liver without altering whole-body sex-differences. Therefore, liver-specific Bcl6-knockout (Bcl6-LKO) mice would be suitable for analyzing sex-biased drug metabolism and pathogenesis of the liver, without needing to consider the effects of whole-body sex-differences.

\section{Materials and methods}

\section{Mice and animal experiments}

Adult and pregnant $\mathrm{C} 57 \mathrm{BL} / 6 \mathrm{~N}$ mice, ICR mice, Balb/c mice, and BDF1 mice were purchased from Nihon SLC (Shizuoka, Japan). Albumin promoter-Cre transgenic mice were obtained from Jackson Laboratory (Bar Harbor, ME) [21, 22]. Bcl6-floxed mice were previously reported and distributed by RIKEN BioResource Research Center (Tsukuba, Japan) [23]. Bcl6-LKO mice were generated by mating the albumin promoter-Cre mouse and Bcl6-floxed mouse. Cre-negative mice with Bcl6-wild-type or floxed alleles were designated as Bcl6-WT mice. Cre-positive mice with the Bcl6-floxed alleles were designated as Bcl6-LKO mice. All animal experiments were performed in accordance with the guidelines. All animal experimental guidelines and protocols were approved by the Institutional Animal Care and Use Committee of Tokai University (Japan, permit number: 173025).

\section{Isolation of fetal, neonatal, and adult livers for expression analysis}

Embryonic day (E) 13, 15, and 17 as well as neonatal livers were excised under the microscope. Adult livers were excised after bleeding out the mice. Total RNA was extracted with TRIzol reagent (Thermo Fisher Scientific, Waltham, MA).

\section{Detection of mRNA by reverse transcription- polymerase chain reaction (RT-PCR)}

First-strand cDNA for quantitative RT-PCR was synthesized using a ReverTra Ace qPCR RT Master Mix with gDNA Remover (TOYOBO, Osaka, Japan) or a HighCapacity cDNA Reverse Transcription Kit (Thermo Fisher Scientific) when RNA was extracted from adult hepatocytes using the RNeasy ${ }^{\circledR}$ Micro Kit (Qiagen, Hilden, Germany). cDNA samples were normalized to the expression of hypoxanthine guanine phosphoribosyl transferase (Hprt). 
Table 1 PCR primers for detection of mouse gene expression

\begin{tabular}{lllr}
\hline Mouse genes & Forward primer $\left(5^{\prime} \rightarrow 3^{\prime}\right)$ & Reverse primer $\left(5^{\prime} \rightarrow 3^{\prime}\right)$ & Probe number \\
Hprt & tcctcctcagaccgctttt & cctggttcatcatcgctaatc & 95 \\
Bc16 & ctgcagatggagcatgttgt & gccatttctgcttcactgg & 4 \\
Cyp2d9 & caggatgctgaaagtactggaac & tcgggaatgcattaagaacc & 106 \\
Cyp2u1 & aaacttttattccttttgggatagg & catcaggaataactccatcttgg & 108 \\
Cyp4a12a/12b & ccactcattcctgcccttc & tcagctcattcatcgcaaac & 63 \\
Cyp7b1 & aattggacagcttggtctgc & ttctcggatgatgctggagt & 99 \\
Cyp2a4/2a5 & accaaggacaccaagtttcg & cagagcccagcataggaaat & 52 \\
Cyp2b9 & agcgccacctccactat & caatctccttttggactttctctg & 15 \\
Cyp2b10 & aagctcattctccagccaga & ctgtgggcaccaggaaag & 106 \\
Cyp2b13 & gcaccactctctgctgtgg & tctccttttggactttctctgc & 15 \\
Cyp3a16 & cttcacaaaccggcagga & tctcttccattcctcatctttagc & 48 \\
Cyp3a41 & ccctgtattgtttggccact & cacagtatcataggtgggaggtg & 4 \\
Cyp3a44 & actcacctgatatccagaagaa & agtatcacaggtgggagttgc & 13 \\
Cyp17a1 & catcccacacaaggctaaca & cagtgcccagagattgatga & 67 \\
ER $\alpha$ & caactgggcaaagagagtgc & ccagacgagaccaatcatca & 19 \\
\hline
\end{tabular}

Quantitative analysis of target mRNA was performed using the Universal Probe Library System (Roche Diagnostics, Basal, Switzerland). Primers and probes used for quantitative RT-PCR are indicated in Table 1.

\section{Isolation of adult hepatocytes}

WT and Bcl6-LKO mice were subjected to a standard twostep collagenase perfusion for isolating primary hepatocytes. The liver was pre-perfused through the portal vein with $0.5 \mathrm{mM}$ EGTA solution and perfused with $0.025 \%$ collagenase (Yakult, Tokyo, Japan) solution. Hepatocytes were purified using $50 \%$ Percoll $^{\mathrm{TM}}$ or Percoll ${ }^{\mathrm{TM}}$ Plus (GE healthcare UK Ltd., Little Chalfont, UK) buffer and then centrifuged at $50 \times g$ for $10 \mathrm{~min}$.

\section{Genomic PCR}

Genomic DNA was purifiedusing the NucleoSpin ${ }^{\circledR}$ Tissue Kit (Macherey-Nagel, Duren, Germany) and used for genomic PCR according to the manufacturer's protocol. To confirm deletion of the Bcl6 allele flanked by loxP sites, PCR was performed using two pairs of primers. The experiment of Genomic PCR1 in Supplementary Figure S1 used the primer pairs of 5'-CCATTCTCAGAAGATTATGGCAGA-3' and 5'CACACTATACATCAGAAAAGAATG-3' [23]. The experiment of Genomic PCR2 in Supplementary Figure S1 used the primer pairs of 5'-CCATTCTCAGAAGATTATGGCAGA-3' and 5'-AGAAAGCCCTCCGGGCAAAGATAG-3'

\section{Western blotting}

Nuclear extracts were prepared from livers or hepatocytes using NE-PER Nuclear and Cytoplasmic Extraction
Reagents (Thermo Fisher Scientific) according to the manufacture's protocol. Nuclear extracts were mixed with SDS sample loading buffer (Wako Pure Chemical Corporation, Osaka, Japan), electrophoresed using a SuperSep $^{\text {TM }}$ Ace $10 \%$ gel (Wako Pure Chemical Corporation), and then electrotransferred onto an Immobilon-P membrane (Millipore, Billerica, MA). The membrane was blocked overnight in phosphate buffered saline (PBS) with Tween 20 (PBS-T) containing $3 \%$ or $5 \%$ Amersham $^{\mathrm{TM}} \mathrm{ECL}^{\mathrm{TM}}$ Blocking Agent (GE healthcare UK Ltd.) and then incubated with primary antibody-Bcl6 antibody (D-8; Santa Cruz Biotechnology, Dallas, TX) - for $2 \mathrm{~h}$. Next, the membrane was washed with PBS-T and incubated with horseradish peroxidase-conjugated secondary antibody (Millipore). During incubation with primary and secondary antibodies, western blot immune booster solutions (Takara Bio Inc., Shiga, Japan) were used to enhance antibody interactions. After another wash with PBS-T, immunoreactive proteins were developed by the ECL reagent (Takara Bio Inc.) and chemiluminescence was captured using the Image Quant LAS 500 (GE healthcare UK Ltd.).

\section{Microarray transcription profile analysis}

For microarray expression analysis, 10-11-week old Bcl6WT and Bcl6-LKO mice of both sexes were dissected and the livers excised after the blood was drained. Total RNA was purified from the livers using TRIzol reagent. Transcriptional profiles were analyzed using the Agilent Whole Mouse Genome Microarray v2 $8 \times 60 \mathrm{~K}$ (Agilent Technologies, Santa Clara, CA). Raw intensity values were normalized using the 75th percentile and transformed to the $\log 2$ scale. Hierarchical clustering of Pearson's correlation coefficients between CYP probe samples was performed using Euclidean 
Fig. 1 Bcl6 expression profiles in the liver. a Expression of Bcl6 in fetal and adult whole livers was analyzed using quantitative PCR. Total RNA was purified from samples derived from embryonic day (E) $13,15,17$ as well as neonatal and 8-week-old adult male livers. Hprt was used as an internal control.

Expression of genes in E13 mouse livers was set to 1.0 . Results are represented as the mean $\pm \mathrm{SD}(n=8$, except for neonatal liver where $n=7)$. $* P$ $<0.05$. b Transcriptional profiles of CYP family genes in Bcl6LKO mice. Adult livers (10-11week-old mice), derived from Bcl6-WT and Bcl6-LKO mice of both sexes, were used for microarray analysis of RNA expression. The probes of putative functional CYPs were extracted from Dr. Nelson's Cytochrome P450 homepage as shown in Method section. We analyzed 138 probes, which are shown in the heat map $(n=4)$
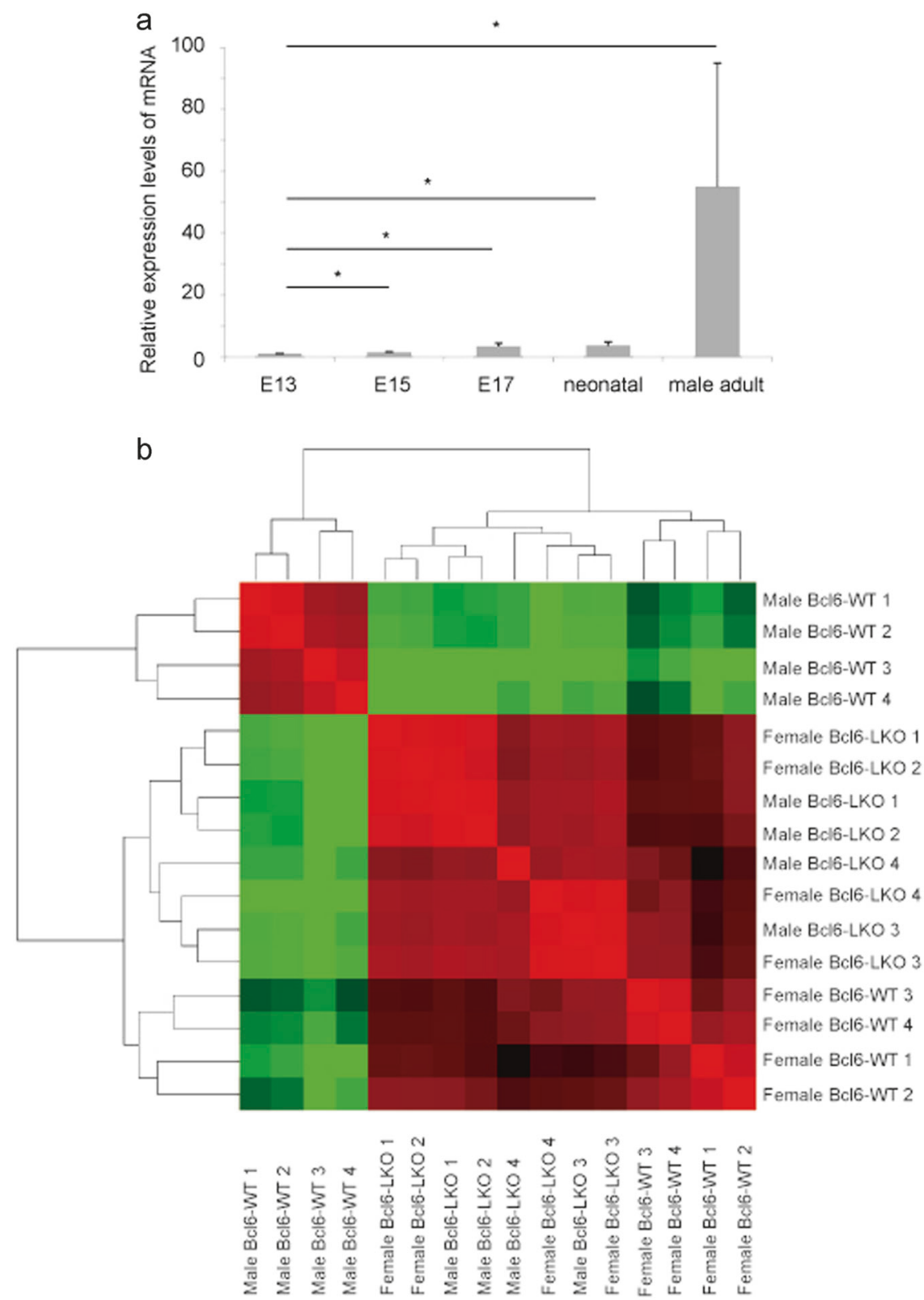

Pearson's correlation coefficients

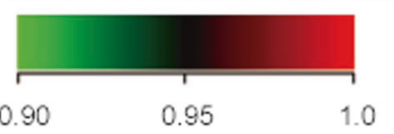

distances and average linkage. Correlations between Bcl6-WT and Bcl6-LKO mice of both sexes were indicated by Pearson's correlation coefficients. CYP probes were extracted in accordance with the Cytochrome P450 database (http:// drnelson.uthsc.edu/mouse.master.table.html). Hierarchical clustering of normalized signal intensities of genes related on signal intensities of sex steroid hormone receptors was performed using Euclidean distances and average linkage. The data has been deposited at the Gene Expression Omnibus (GSE89091 and GSE107435).

\section{Measurement of CYP2B activity}

Purified adult hepatocytes were inoculated on collagen-1-coated dishes in William's medium E (Sigma-Aldrich, St. Louis, MO) supplemented with $10 \%$ fetal bovine serum (FBS, Nichirei Biosciences, Tokyo, Japan), $1 \times$ insulin-transferrin-selenium $\mathrm{X}$ (Thermo Fisher Scientific), $10^{-7} \mathrm{M}$ dexamethasone, and $1 \times$ penicillin-streptomycin-glutamine (Thermo Fisher Scientific). After adhesion, the cells were gently washed with PBS and the CYP2B substrate luciferin-2B6 was added and incubated for $1 \mathrm{~h}$. 
Fig. 2 Expression of maledependent CYP mRNA in male and female Bcl6-LKO mouse livers. Total RNA was purified from samples derived from 8-11-week-old mice. The expression levels of Cyp2d9, $2 \mathrm{u} 1,4 \mathrm{a} 12 \mathrm{a} / 12 \mathrm{~b}$, and $7 \mathrm{~b} 1 \mathrm{mRNA}$ were analyzed using quantitative PCR. Hprt was used as an internal control. Expression of genes in male Bcl6-WT mouse livers was set to 1.0. Results are represented as the mean $\pm \mathrm{SD}(n$ $=10$ for male wild-type mice, $n$ $=8$ for female wild-type mice, $n=9$ for male Bcl6-LKO mice, and $n=7$ for female Bcl6-LKO mice). $* P<0.05$. MWT, male wild-type mouse samples; FWT, female wild-type mouse samples; MLKO, male liverspecific knockout mouse samples; FLKO, female liverspecific knockout mouse samples

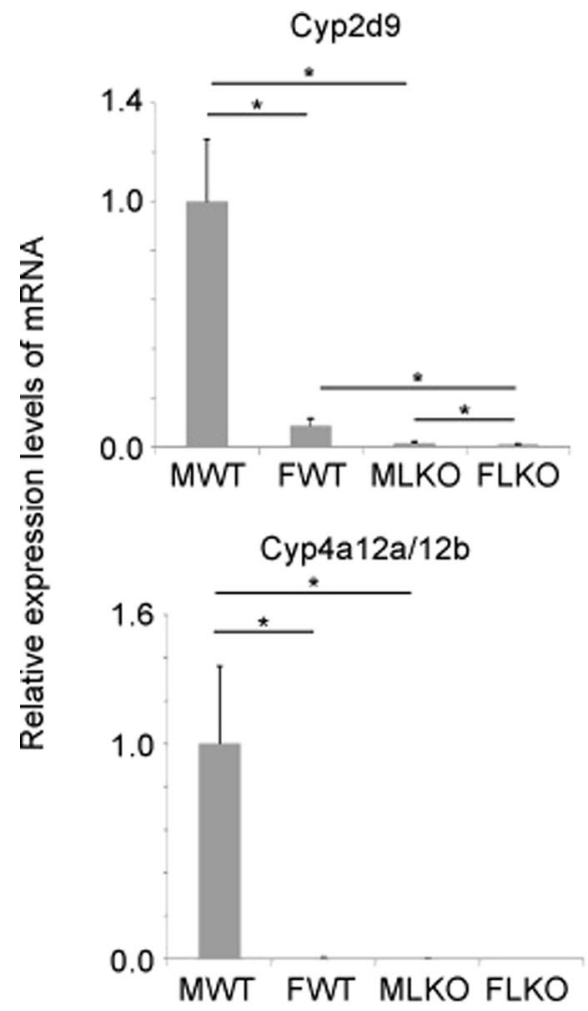

CYP2B activity was measured using the P450-Glo ${ }^{\mathrm{TM}}$ CYP2B6 Assay (Promega, Madison, WI) according to the manufacturer's protocol. The amount of total protein was used to correct the luminescence.

\section{Measurement of sex steroid hormones in serum}

The serum from WT and Bcl6-LKO mice was obtained from the heart and then sex steroid hormone levels ( $\beta$ estradiol and testosterone) were analyzed using liquid chromatography-tandem mass spectrometry by ASKA Pharmamedical Co., Ltd. (Kawasaki, Japan).

\section{Isolation of adipose tissue for expression analysis}

Bcl6-WT and Bcl6-LKO mice of both sexes were dissected and then adipose tissue was excised after the blood was drained. Total RNA was purified from samples using the RNeasy Lipid Tissue Mini Kit (Qiagen) according to the manufacturer's protocol.

\section{Statistics}

Microsoft Excel 2010 (Microsoft, Redmond, WA) was used to calculate standard deviation (SD) and statistically significant differences between samples using a two-tailed Student's $t$-test

\section{Results}

\section{Establishment of liver-specific Bcl6-knockout mice for the analysis of hepatocytic Bcl6 functions}

A network of transcription factors are expressed during liver development to regulate hepatoblast maturation [24]. We have previously analyzed the changes in transcription factor expression patterns between mid-fetal and adult liver development using microarrays and have identified several candidate transcription factors that may regulate hepatocyte functions. For example, Mist1 was transiently upregulated in perinatal livers and is involved in hepatoblast maturation [25]. In addition, expression of Bcl6, another candidate gene, increased during hepatocytic maturation, as measured by quantitative PCR (Fig. 1a). The phenotypes of Bcl6knockout mice have been previously reported [18, 19]. Until the perinatal stage, Bcl6-knockout homozygous mice were found in a 1:2:1 Mendelian ratio; however, these homozygous mice were weak and most died within 5 weeks of birth. In order to evaluate hepatocytic Bcl6 function in adult mouse livers, we generated Bcl6-LKO mice by crossing Bcl6-floxed mice [23] with albumin promoter-Cre transgenic mice, which express Cre recombinase in hepatocytes [21, 22]. We confirmed the deletion of Bcl6 in hepatocytes derived from Cre-positive mice with Bcl6floxed alleles (Supplementary Figure S1). Cre-negative mice were designated as Bcl6-WT mice and Cre-positive 
Fig. 3 Expression of femaledependent CYP mRNA in male and female Bcl-LKO mouse livers. The expression levels of Cyp2a4/2a5, 2b9, 2b10, 2b13, $3 \mathrm{a} 16,3 \mathrm{a} 41,3 \mathrm{a} 44$, and $17 \mathrm{a} 1$ mRNA were analyzed using quantitative PCR. Hprt was used as an internal control.

Expression of genes in female Bcl6-WT mouse livers was set to 1.0. Results are represented as the mean $\pm \mathrm{SD}(n=10$ for male wild-type mice, $n=8$ for female wild-type mice, $n=9$ for male Bcl6-LKO mice, and $n=7$ for female Bcl6-LKO mice). $* P<$ 0.05 . MWT, male wild-type mouse samples; FWT, female wild-type mouse samples; MLKO, male liver-specific knockout mouse samples; FLKO, female liver-specific knockout mouse samples, N.D. not determined

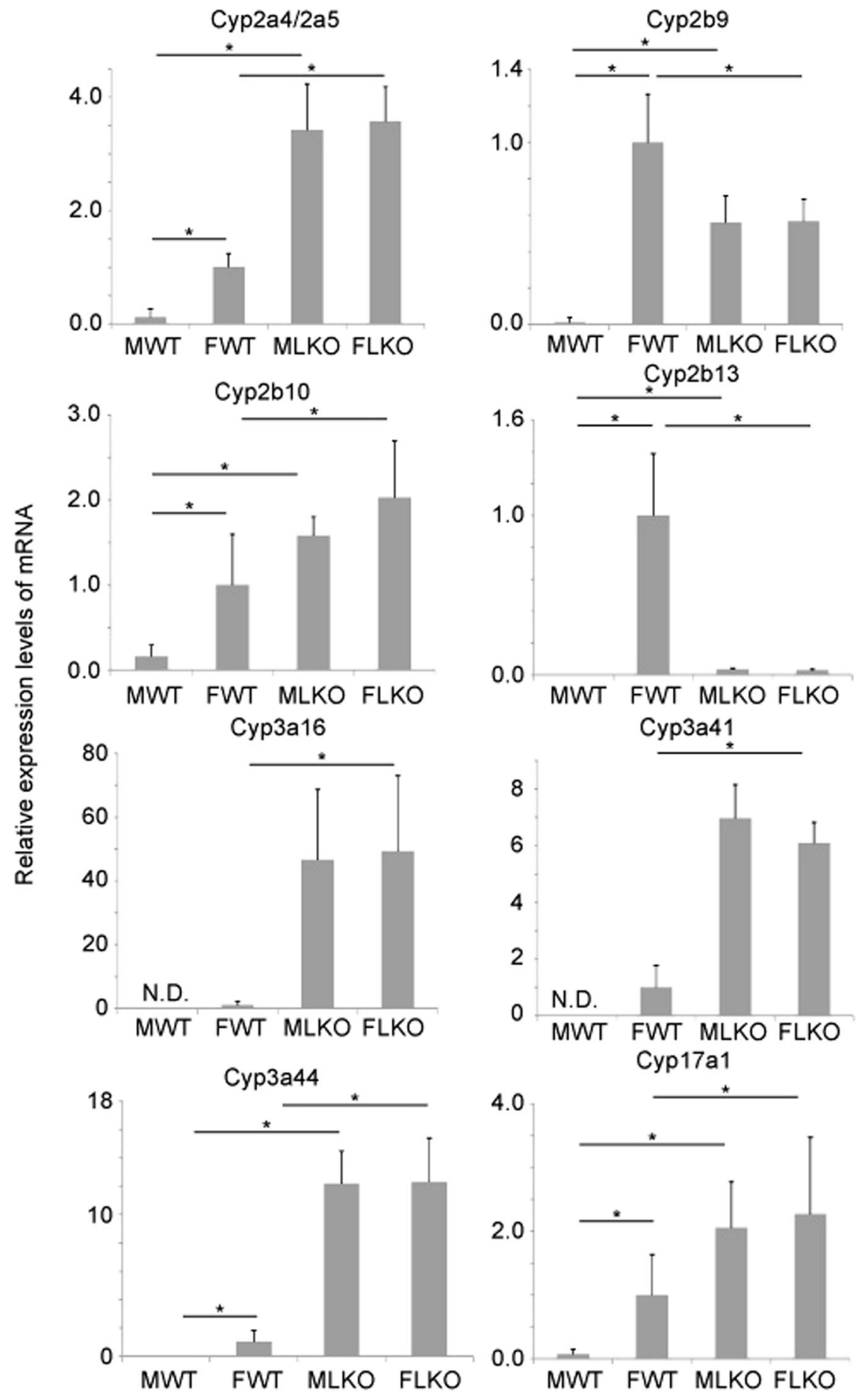

mice with the Bcl6-floxed allele as Bcl6-LKO mice in the following experiments.

\section{Gene expression profiles of liver-specific Bcl6- knockout mouse livers}

To investigate $\mathrm{Bcl} 6$ function in the adult liver, microarray analyses was performed using Bcl6-LKO mouse livers. We compared expressional profiles of CYP drug metabolic enzyme families between male and female livers. Sex biases in drug metabolism have been shown in mice [4, 5, 26-28], rats $[29,30]$, and humans $[6,7,31]$ and are due to sexdependent gene regulation [32-34]. We extracted over 100 putative functional CYPs by eliminating pseudogenes. Figure 1b shows the expression profiles of CYPs in Bcl6WT and KO livers of both sexes. The Pearson's correlation 
coefficient was used to indicate correlation between gene expression profiles among the samples. Between the same sex livers of Bcl6-WT mice, the correlation coefficient was almost 1.0. The correlation coefficient was low between male and female livers of Bcl6-WT mice, suggesting that the sex-differential expression profiles of CYPs were maintained in normal mice. In contrast, sex-differential CYP expression was abolished in the livers of male and female Bcl6-LKO mice. Interestingly, the expression profiles of both male and female Bcl6-LKO mice livers were similar to those of female Bcl6-WT mice. These findings imply that Bcl6 is more active in male livers in regulating the expression of CYPs. Thus, we theorized that sex-related differences in drug metabolism is regulated by hepatocytic Bc16.

\section{Gene expression profiles of drug metabolic genes in liver-specific Bcl6-knockout mouse livers}

Next, we examined several sex-differential CYP expression profiles by quantitative PCR. Expression of Cyp2d9, 2u1, $4 \mathrm{a} 12 \mathrm{a} / 12 \mathrm{~b}$, and $7 \mathrm{~b} 1$ in normal male livers was significantly higher than in female livers. In contrast, these male-biased genes were downregulated in the livers of male Bcl6-LKO mice (Fig. 2). Moreover, expression of Cyp2a4/2a5, 2b9, 2b10, 2b13, 3a16, 3a41, 3a44, and 17a1 in normal male livers was significantly lower than that in the female livers. These female-biased genes were upregulated in the livers of male Bcl6-LKO mice (Fig. 3). In addition, the differences in CYP expression patterns between male and female livers were abolished in Bcl6-LKO mice (Figs. 2 and 3). These results indicate that Bcl6 is an important regulator of sexdifferential expression of CYPs. In particular, it appears that Bcl6 is mainly functional in male livers to control malebiased CYP expression profiles.

To eliminate the possibility of genetic background specificity regarding sex-biased gene expression of CYPs, we also examined CYP expression patterns in other mouse strains including ICR, Balb/c, and BDF1. Expression of the male-biased genes Cyp2d9 and $4 \mathrm{a} 12 \mathrm{a} / 12 \mathrm{~b}$ was found higher in male livers than in female livers of ICR, Balb/c, and BDF1 mice, which was the same pattern observed in C57B6 mice (Supplementary Figure S2). Expression of the femalebiased genes Cyp2a4/2a5, 2b9, 3a41, and 17a1 was also higher in female livers than in male livers of the three mouse strains (Supplementary Figure S3). These results suggest that sex-biased gene expression of CYPs is independent of mouse strain.

\section{Measurement of CYP2B activity in adult hepatocytes}

Several Cyp2b genes were induced in Bcl6-WT female livers, as indicated above. We therefore measured CYP2B a Isolation and culture of hepatocytes on collagen coated dishes
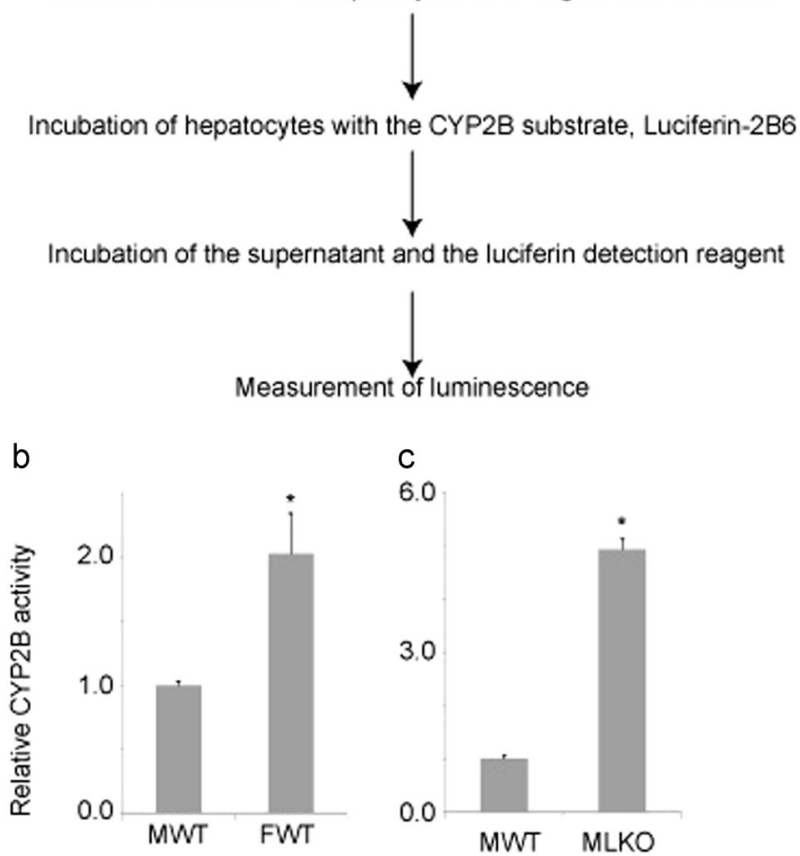

Fig. 4 Measurement of CYP2B activity in adult hepatocytes. a The schematic for measuring CYP2B activity in adult hepatocytes. b Female-biased CYP2B activity in male and female Bcl6-WT hepatocytes. c Regulation of CYP2B activity by Bcl6. The total protein amount was used to correct for CYP2B activity. CYP2B activity of male WT was set to 1.0. The results are represented as the mean relative $\mathrm{CYP} 2 \mathrm{~B}$ activity $\pm \mathrm{SD}(n=6)$. ${ }^{*} P<0.05$. The representative data were shown. The experiments were independently repeated at least three times. The results of the other experiments were shown in Supplementary Figure S4. MWT, male wild-type mouse samples; FWT, female wild-type mouse samples; MLKO, male liver-specific knockout mouse samples

activity to confirm whether changes in gene expression are reflected in enzymatic activity. CYP2B activity in wild-type female hepatocytes was higher than that in wild-type male hepatocytes. Moreover, CYP2B activity in male hepatocytes was induced by Bcl6 deletion (Fig. 4 and Supplementary Figure S4). These changes in CYP2B activity were consistent with the changes in mRNA expression, indicating that Bcl6 regulates the enzymatic activity of CYP2B through regulation of mRNA expression patterns.

\section{Assessment of sex steroid hormone levels and reproductive abilities}

Androgen and estrogen are sex-dependent hormones that regulate the development and maintenance of male and female characteristics. We thus analyzed whether deletion of hepatocytic Bcl6 directly regulates sex-biased drug metabolism in the liver, as Bcl6 may regulate liver drug metabolism through the control of sex-dependent hormone production. The serum protein levels of sex steroid hormones were analyzed using liquid chromatography-tandem 


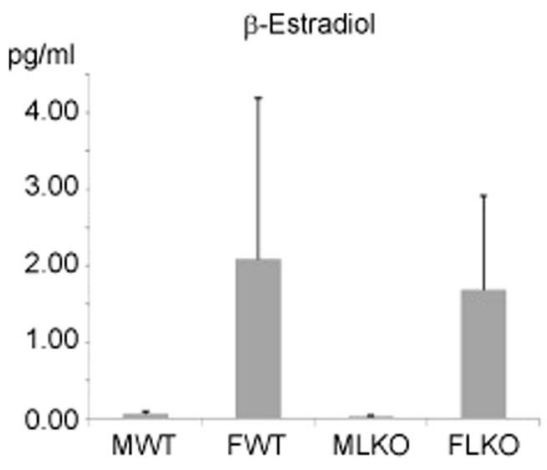

Fig. 5 Measurement of sex steroid hormone levels in serum. Sex steroid hormones ( $\beta$-estradiol and testosterone) in the serum were analyzed by liquid chromatography-tandem mass spectrometry $(n=$

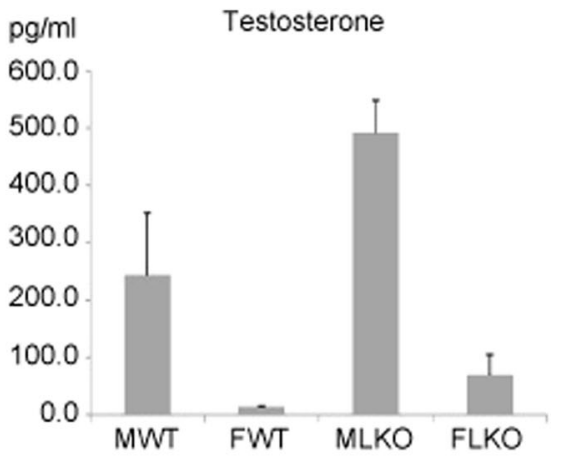

3). MWT, male wild-type mouse samples; FWT, female wild-type mouse samples; MLKO, male liver-specific knockout mouse samples; FLKO, female liver-specific knockout mouse samples

Table 2 Mating results

\begin{tabular}{|c|c|c|c|c|c|}
\hline \multirow[t]{2}{*}{ Male } & \multirow[t]{2}{*}{ Female } & \multicolumn{4}{|c|}{ Resulting genotype } \\
\hline & & Male Bcl6 $6^{\text {lox/lox }}$ & Male Alb-Cre + Bcl6 $6^{\text {lox/lox }}$ & Female Bcl6 $6^{\text {lox/lox }}$ & Female Alb-Cre + Bcl6 $6^{\text {lox/lox }}$ \\
\hline $\mathrm{Bcl} 6^{\mathrm{lox} / \mathrm{lox}}$ & Alb-Cre + Bcl6 $6^{\text {lox/lox }}$ & 60 & 52 & 45 & 50 \\
\hline $\mathrm{Alb}-\mathrm{Cre}+\mathrm{Bcl} 6^{\mathrm{lox} / \mathrm{lox}}$ & $\mathrm{Bcl} 6^{\mathrm{lox} / \mathrm{lox}}$ & 36 & 44 & 30 & 39 \\
\hline
\end{tabular}

mass spectrometry. Levels of the major androgen (testosterone) and the most active estrogen ( $\beta$-estradiol) in serum were measured. Testosterone levels were higher in males than in females of both WT and Bcl6-LKO mice and $\beta$ estradiol levels were higher in females than in males of both WT and Bcl6-LKO mice (Fig. 5), suggesting that deletion of hepatocytic Bcl6 did not alter sex-dependent hormone production. In addition, Bcl6-LKO male and female mice exhibited normal reproductive abilities (Table 2).

\section{Gene expression of sex steroid hormone receptors in liver-specific Bcl6-knockout mouse livers}

As mentioned above, ligand expression of sex steroid hormones did not change after Bcl6 hepatocytic deletion. Thus, we next analyzed gene expression of sex steroid hormone receptor signaling molecules using a microarray and found that expression of estrogen receptor $\alpha(\mathrm{ER} \alpha)$ was induced in livers derived from both male and female Bcl6-LKO mice (Fig. 6a). We then confirmed the expression of ER $\alpha$ in liver and non-liver tissues using quantitative PCR (Fig. 6b). The expression levels of the female hormone receptor $\mathrm{ER} \alpha$ in Bcl6-WT female livers were higher than in Bcl6-WT male livers. This female-biased expression in ER $\alpha$ was also confirmed in ICR, Balb/c, and BDF1 mice (Supplementary Figure S5). Hepatocytic Bcl6 deletion induced expression of ER $\alpha$ in both male and female mouse livers. In contrast to the liver, expression levels of $\mathrm{ER} \alpha$ did not change in adipose tissues derived from Bcl6-LKO mice (Fig. 6b). These results indicate that Bcl6 deletion in hepatocytes may regulate liver-specific sex-dependent characteristics by altering the expression levels of sex steroid hormone receptors in the liver.

\section{Production of Bcl6 in male and female hepatocytes}

In rat livers, production of Bcl6 is regulated by sex-specific expression patterns of $\mathrm{GH}$ and female livers were shown to have less Bcl6 proteins than that of male livers [16]. We thus evaluated the production of Bcl6 in male and female adult mouse hepatocytes. Bcl6 mRNA was detected in hepatocytes derived from both male and female wild-type mice, but expression levels did not differ between them (Fig. 7a). Next, we analyzed expression patterns of the Bcl6 protein in nuclei using western blot analysis. The specificity of the Bcl6 antibody was confirmed as shown in Supplementary Figure S6. Bcl6 protein was not detected in Bcl6LKO mouse livers. The protein expression levels of Bcl6 were not significantly different between male and female hepatocytes in Bcl6-WT mice (Fig. 7b). These results suggest that the molecular mechanism regulating sexdependent activity of Bcl6 in hepatocytes is different among species.

\section{Discussion}

This study is the first to analyze Bcl6 function in hepatic sexdependent gene expression using liver-specific Bcl6-knockout 
Fig. 6 Expression of sex steroid hormone receptors in male and female Bcl6-LKO mouse liver and other tissues. a

Transcriptional profiles of androgen- and estrogen-related genes. ER $\alpha$ probes (Esr1, indicated by arrowheads) were upregulated in both male and female livers derived from Bcl6LKO mice $(n=4)$. b Expression levels of ER $\alpha$ in the liver and adipose tissue. Hprt was used as an internal control. Expression of genes in male Bcl6-WT mice was set to 1.0. Results are represented as the mean $\pm \mathrm{SD}$ ( $n$ $=6$ for male wild-type mice, $n$ $=5$ for female wild-type mice, $n=5$ for male Bcl6-LKO mice, and $n=4$ for female Bcl6-LKO mice). $* P<0.05$. MWT, male wild-type mouse samples; FWT, female wild-type mouse samples; MLKO, male liverspecific knockout mouse samples; FLKO, female liverspecific knockout mouse samples
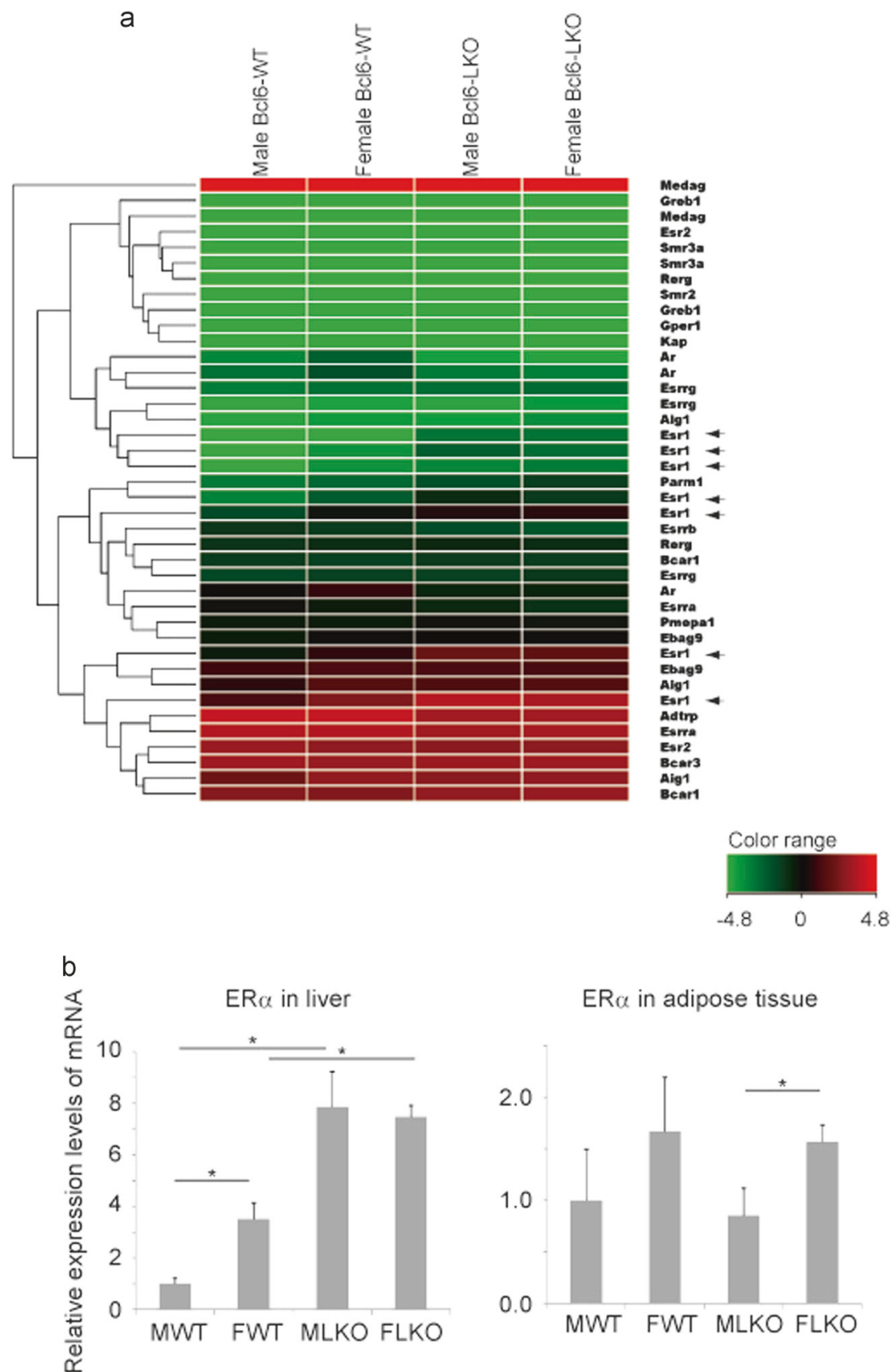

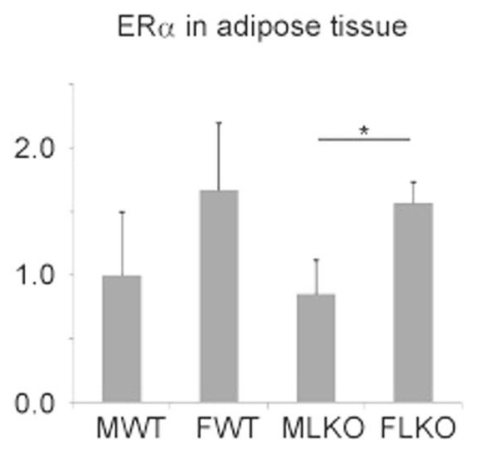

mice. Bcl6 was found important for regulating sex-differential expression of CYPs in the male liver. In male Bcl6-LKO mice, the expression of male-biased CYPs was downregulated, whereas the expression of female-biased CYPs was upregulated, meaning that the sex-biased expression profiles of CYPs were not maintained between male and female livers derived from Bcl6-LKO mice. These results suggest that hepatocytic Bcl6 is a key factor in maintaining male-biased drug metabolism in the liver. The difference in Bcl6 protein levels is predominant between male and female livers in rats [16]. Plasma GH is dimorphic between males and females and GH secretion controls sex-biased Bcl6 expression in the rat liver. In contrast, we found that protein levels of hepatocytic Bcl6 were similar between both male and female mouse livers in this study. One of the reasons for this observation is that plasma GH profiles are extremely sex-differential in rats [35] yet milder in mice [36]. As shown above, Bcl6 expression in mice hepatocytes is required for sex-biased gene expression in male livers. Thus, we theorized that Bcl6 DNA-binding activities in the wild-type male mice are higher than that in female mice. The high induction of some female-type CYP expression levels, such as CYP3a16, 41, and 44, in Bcl6LKO mice, may be due to the complete suppression of Bcl6 activity. Our future studies will focus on analyzing about the 


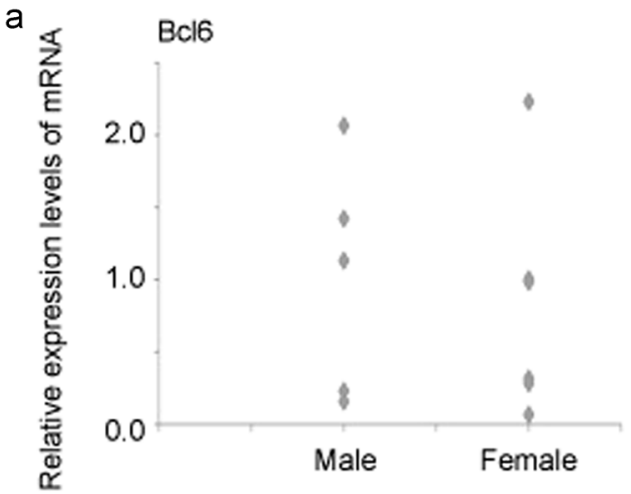

b

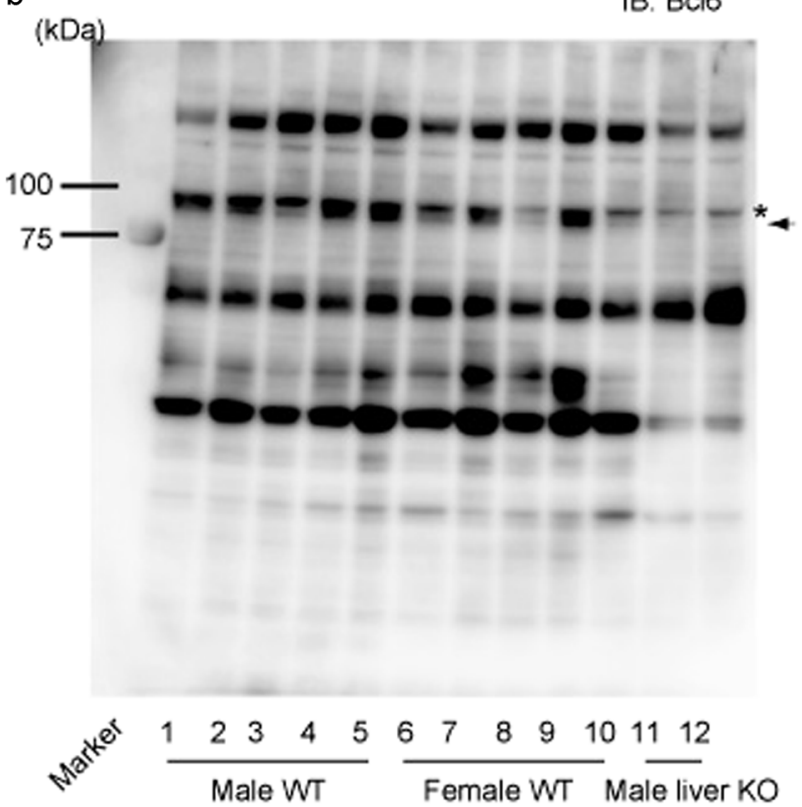

Fig. 7 Assessment of Bcl6 mRNA expression and protein levels between male- and female-derived hepatocytes. a Total RNA was purified from 8-week-old male and female murine hepatocytes. Bcl6 expression was analyzed by quantitative PCR. Hprt was used as an internal control ( $n=5$ for males and $n=6$ for females). b Nuclear extracts were prepared from male and female murine hepatocytes. Bcl6 protein levels were analyzed by western blotting $(n=5)$. The arrowhead indicates the specific Bcl6 protein shown in Supplementary Figure S6. The asterisk indicates a non-specific signal

Bcl6 DNA-binding abilities and genes that are directly regulated by Bcl6.

Blimp1 is a Bcl6-regulated transcriptional repressor that functions as a Bcl6 antagonist in immune cells [37]. The balance between Bcl6 and Blimp1 expression is important for immune system development. In microarray expression analysis, Blimp1 expression barely changed in male Bcl6LKO mouse livers, suggesting that Blimp1 in the liver is not directly regulated by hepatocytic Bcl6. Cux2 is a femalebiased transcription factor in the liver that is regulated by Bcl6 through Cux 2 promoter regions in the liver and other tissues $[16,38]$. We found that expression of Cux2 was significantly upregulated after hepatocytic Bcl6 deletion in males using microarray analysis, indicating that Bcl6 may regulate liver functions through its downstream gene, Cux2. Other factors may also be involved in the regulation of sexdifferential CYP expression. In immune cells, Bcl6 controls expression of its indirect target genes through miRNA expression regulation [39-41]. In the drug metabolic system, the untranslated region of some CYPs contain miRNA binding sequences [42, 43]. Thus, miRNAs may be direct targets of Bcl6 during sex-dependent regulation of metabolic genes in the liver. Another possibility is that sexdifferential CYPs are directly regulated by hepatocytic Bcl6 through CYP promoter and enhancer regions. In B cells, Bcl6 interacts with transcriptional corepressors such as HDAC, NCoR, SMRT [44], MTA3 [45], BCoR [46], and histone methyltransferase Ezh2 [47]. Bcl6 also interacts with the transcription factor Miz-1 and represses Miz-1 target genes independent of the Bcl6 binding sequence [48]. Acetylation of Bcl6 by p300 inhibits Bcl6 as a transcriptional repressor in B cells [49]. Therefore, Bcl6 acts as a transcriptional repressor by interacting with other corepressors to regulate gene expression through a complex transcriptional network. As such, several direct target genes have already been reported in germinal center B cells [37, 50-52]. In most cases, Bcl6 represses the expression of its target genes in a DNA sequence-specific manner. Thus, these target genes may be important for the regulation of sex-biased drug metabolic enzymes by hepatocytic Bcl6.

Deletion of Bcl6 altered liver sex-related differences but not overall body sex-related differences as serum levels of sex hormones and mice reproductive ability did not change. Bcl6 deletion in hepatocytes induced expression of $\mathrm{ER} \alpha$ in both male and female livers and thus sex-steroid hormone signaling may be regulated by Bcl6 through upregulation of the estrogen receptor; however, production of the estradiol ligand did not significantly change after hepatocytic Bcl6 deletion. In addition, induction of $\mathrm{ER} \alpha$ expression in Bcl6LKO mice was detected in the liver, but not in non-liver tissues (adipose tissue). These results suggest that hepatocytic Bcl6 expression is important for sex-biased, drug metabolic gene expression in the liver and that our model is useful for analyzing liver-specific sex-dependent drug metabolism. Liver metabolism by hepatocytic CYPs is very important for the pharmacokinetics of several therapeutic medicines. Therefore, the pharmacokinetics of candidate molecules must be evaluated in experimental animal models and human liver samples during drug discovery. As shown above, drug metabolism in the liver is different between males and females due to sex-biased CYP expression. Male and female-related hormones regulate not only hepatocytic sex-related differences but also sex-related differences of other tissues (e.g., reproductive and endocrine organs). It is possible that sex-dependent characteristics of other tissues 
affects the physiology of experimental animals supplied with medicines during drug discovery. In addition, the frequency of several liver diseases, such as hepatocellular carcinomas (HCC), is different between males and females. There is a marked male predominance in morbidity and mortality of HCC patients and it has been shown that the sex-dependent hormone estrogen has a protective effect against HCC progression [53]. However, the correlation between HCC pathology and sex-related differences in the liver remains unknown. This report is the first study to establish a mouse model regulating sex-based CYP expression in the liver without affecting the sex of the whole body. Previously, several studies have established chimera mice with human hepatocytes using immunodeficient mice. The humanized hepatic mouse models having hepatocytic-specific Bcl6 deletion may be useful for drug discovery and pathology regulated by sex-biased characteristics in humans.

Acknowledgements Bcl6-floxed mice were kindly provided by Prof. Toshitada Takemori at the Research Center for Allergy and Immunology, RIKEN (Yokohama, Japan). Some analyses were performed by the Support Center for Medical Research and Education, Tokai University (Isehara, Japan). This study was supported in part by a Grant-in-Aid for Scientific Research from the Ministry of Education, Culture, Sports, and Technology, Japan (26293178 and 16K15434 to AK; 16K19060 to HC). This study was also supported in part by the Research and Study Project of Tokai University Educational System General Research Organization (to AK) and Tokai University School of Medicine Research Aid (to HC).

\section{Compliance with ethical standards}

Conflict of interest The authors declare that they have no conflict of interest.

\section{References}

1. Rinn JL, Snyder M. Sexual dimorphism in mammalian gene expression. Trends Genet. 2005;21:298-305.

2. Nelson DR, Zeldin DC, Hoffman SM, et al. Comparison of cytochrome P450 (CYP) genes from the mouse and human genomes, including nomenclature recommendations for genes, pseudogenes and alternative-splice variants. Pharmacogenetics. 2004; 14:1-18.

3. Renaud HJ, Cui JY, Khan M, et al. Tissue distribution and genderdivergent expression of 78 cytochrome P450 mRNAs in mice. Toxicol Sci. 2011;124:261-77.

4. Jarukamjorn K, Sakuma T, Jaruchotikamol A, et al. Regulation of mouse hepatic CYP2D9 mRNA expression by growth and adrenal hormones. Drug Metab Pharmacokinet. 2006;21:29-36.

5. Sakuma T, Takai M, Endo Y, et al. A novel female-specific member of the CYP3A gene subfamily in the mouse liver. Arch Biochem Biophys. 2000;377:153-62.

6. Hunt CM, Westerkam WR, Stave GM. Effect of age and gender on the activity of human hepatic CYP3A. Biochem Pharmacol. 1992;44:275-83.

7. Lew KH, Ludwig EA, Milad MA, et al. Gender-based effects on methylprednisolone pharmacokinetics and pharmacodynamics. Clin Pharmacol Ther. 1993;54:402-14.
8. Waxman DJ, O'Connor C. Growth hormone regulation of sexdependent liver gene expression. Mol Endocrinol. 2006;20: 2613-29.

9. Holloway MG, Laz EV, Waxman DJ. Codependence of growth hormone-responsive, sexually dimorphic hepatic gene expression on signal transducer and activator of transcription $5 \mathrm{~b}$ and hepatic nuclear factor 4alpha. Mol Endocrinol. 2006;20:647-60.

10. Waxman DJ, Ram PA, Park SH, et al. Intermittent plasma growth hormone triggers tyrosine phosphorylation and nuclear translocation of a liver-expressed, Stat 5-related DNA binding protein. Proposed role as an intracellular regulator of male-specific liver gene transcription. J Biol Chem. 1995;270:13262-70.

11. Clodfelter KH, Holloway MG, Hodor P, et al. Sex-dependent liver gene expression is extensive and largely dependent upon signal transducer and activator of transcription 5b (STAT5b): STAT5bdependent activation of male genes and repression of female genes revealed by microarray analysis. Mol Endocrinol. 2006;20:1333-51.

12. Udy GB, Towers RP, Snell RG, et al. Requirement of STAT5b for sexual dimorphism of body growth rates and liver gene expression. Proc Natl Acad Sci USA. 1997;94:7239-44.

13. Lo Coco F, Ye BH, Lista F, et al. Rearrangements of the BCL6 gene in diffuse large cell non-Hodgkin's lymphoma. Blood. 1994; 83:1757-9.

14. Kawamata N, Miki T, Ohashi K, et al. Recognition DNA sequence of a novel putative transcription factor, BCL6. Biochem Biophys Res Commun. 1994;204:366-74.

15. Chang CC, Ye BH, Chaganti RS, et al. BCL-6, a POZ/zinc-finger protein, is a sequence-specific transcriptional repressor. Proc Natl Acad Sci USA. 1996;93:6947-52.

16. Meyer RD, Laz EV, Su T, et al. Male-specific hepatic Bcl6: growth hormone-induced block of transcription elongation in females and binding to target genes inversely coordinated with STAT5. Mol Endocrinol. 2009;23:1914-26.

17. Zhang Y, Laz EV, Waxman DJ. Dynamic, sex-differential STAT5 and BCL6 binding to sex-biased, growth hormone-regulated genes in adult mouse liver. Mol Cell Biol. 2012;32:880-96.

18. Dent AL, Shaffer AL, Yu X, et al. Control of inflammation, cytokine expression, and germinal center formation by BCL-6. Science. 1997;276:589-92.

19. Yoshida T, Fukuda T, Hatano M, et al. The role of Bcl6 in mature cardiac myocytes. Cardiovasc Res. 1999;42:670-9.

20. LaPensee CR, Lin G, Dent AL, et al. Deficiency of the transcriptional repressor B cell lymphoma 6 (Bc16) is accompanied by dysregulated lipid metabolism. PLoS ONE. 2014;9:e97090.

21. Postic C, Shiota M, Niswender KD, et al. Dual roles for glucokinase in glucose homeostasis as determined by liver and pancreatic beta cell-specific gene knock-outs using Cre recombinase. J Biol Chem. 1999;274:305-15.

22. Postic C, Magnuson MA. DNA excision in liver by an albuminCre transgene occurs progressively with age. Genesis. 2000;26: $149-50$.

23. Kaji T, Ishige A, Hikida M, et al. Distinct cellular pathways select germline-encoded and somatically mutated antibodies into immunological memory. J Exp Med. 2012;209:2079-97.

24. Lemaigre FP. Mechanisms of liver development: concepts for understanding liver disorders and design of novel therapies. Gastroenterology. 2009;137:62-79.

25. Chikada H, Ito K, Yanagida A, et al. The basic helix-loop-helix transcription factor, Mist1, induces maturation of mouse fetal hepatoblasts. Sci Rep. 2015;5:14989.

26. Jarukamjorn K, Sakuma T, Jaruchotikamol A, et al. Modified expression of cytochrome P450 mRNAs by growth hormone in mouse liver. Toxicology. 2006;219:97-105.

27. Wauthier V, Sugathan A, Meyer RD, et al. Intrinsic sex differences in the early growth hormone responsiveness of sex-specific genes in mouse liver. Mol Endocrinol. 2010;24:667-78. 
28. Conforto TL, Waxman DJ. Sex-specific mouse liver gene expression: genome-wide analysis of developmental changes from pre-pubertal period to young adulthood. Biol Sex Differ. 2012;3:9.

29. Ahluwalia A, Clodfelter KH, Waxman DJ. Sexual dimorphism of rat liver gene expression: regulatory role of growth hormone revealed by deoxyribonucleic acid microarray analysis. Mol Endocrinol. 2004;18:747-60.

30. Wauthier V, Waxman DJ. Sex-specific early growth hormone response genes in rat liver. Mol Endocrinol. 2008;22:1962-74.

31. Wolbold R, Klein K, Burk O, et al. Sex is a major determinant of CYP3A4 expression in human liver. Hepatology. 2003;38: 978-88.

32. Yokomori N, Kobayashi R, Moore R, et al. A DNA methylation site in the male-specific P450 (Cyp 2d-9) promoter and binding of the heteromeric transcription factor GABP. Mol Cell Biol. 1995;15:5355-62.

33. Waxman DJ, Celenza JL. Sexual dimorphism of hepatic gene expression: novel biological role of KRAB zinc finger repressors revealed. Genes Dev. 2003;17:2607-13.

34. Bhadhprasit W, Sakuma T, Kawasaki Y, et al. Hepatocyte nuclear factor 4alpha regulates expression of the mouse female-specific Cyp3a41 gene in the liver. Drug Metab Dispos. 2011;39:490-7.

35. Jansson JO, Eden S, Isaksson O. Sexual dimorphism in the control of growth hormone secretion. Endocr Rev. 1985;6:128-50.

36. MacLeod JN, Pampori NA, Shapiro BH. Sex differences in the ultradian pattern of plasma growth hormone concentrations in mice. J Endocrinol. 1991;131:395-9.

37. Tunyaplin C, Shaffer AL, Angelin-Duclos CD, et al. Direct repression of prdm 1 by Bcl-6 inhibits plasmacytic differentiation. J Immunol. 2004;173:1158-65.

38. Laz EV, Holloway MG, Chen CS, et al. Characterization of three growth hormone-responsive transcription factors preferentially expressed in adult female liver. Endocrinology. 2007;148: 3327-37.

39. Yu D, Rao S, Tsai LM, et al. The transcriptional repressor Bcl-6 directs $\mathrm{T}$ follicular helper cell lineage commitment. Immunity. 2009;31:457-68.

40. Basso K, Schneider C, Shen Q, et al. BCL6 positively regulates AID and germinal center gene expression via repression of miR155. J Exp Med. 2012;209:2455-65.
41. Sawant DV, Wu H, Kaplan MH, et al. The Bcl6 target gene microRNA-21 promotes Th2 differentiation by a $\mathrm{T}$ cell intrinsic pathway. Mol Immunol. 2013;54:435-42.

42. Xie X, Miao L, Yao J, et al. Role of multiple microRNAs in the sexually dimorphic expression of Cyp2b9 in mouse liver. Drug Metab Dispos. 2013;41:1732-7.

43. Singh D, Kashyap A, Pandey RV, et al. Novel advances in cytochrome P450 research. Drug Discov Today. 2011;16: 793-9.

44. Huynh KD, Bardwell VJ, TheBCL-6 POZ domain and other POZ domains interact with the co-repressors $\mathrm{N}-\mathrm{CoR}$ and SMRT. Oncogene. 1998;17:2473-84.

45. Fujita N, Jaye DL, Geigerman C, et al. MTA3 and the Mi-2/NuRD complex regulate cell fate during B lymphocyte differentiation. Cell. 2004;119:75-86.

46. Huynh KD, Fischle W, Verdin E, et al. BCoR, a novel corepressor involved in BCL-6 repression. Genes Dev. 2000;14:1810-23.

47. Beguelin W, Teater M, Gearhart MD, et al. EZH2 and BCL6 cooperate to assemble CBX8-BCOR complex to repress bivalent promoters, mediate germinal center formation and lymphomagenesis. Cancer Cell. 2016;30:197-213.

48. Phan RT, Saito M, Basso K, et al. BCL6 interacts with the transcription factor Miz-1 to suppress the cyclin-dependent kinase inhibitor p21 and cell cycle arrest in germinal center B cells. Nat Immunol. 2005;6:1054-60.

49. Bereshchenko OR, Gu W, Dalla-Favera R. Acetylation inactivates the transcriptional repressor BCL6. Nat Genet. 2002;32:606-13.

50. Phan RT, Dalla-Favera R. The BCL6 proto-oncogene suppresses p53 expression in germinal-centre B cells. Nature. 2004;432: $635-9$.

51. Ranuncolo SM, Polo JM, Dierov J, et al. Bcl-6 mediates the germinal center B cell phenotype and lymphomagenesis through transcriptional repression of the DNA-damage sensor ATR. Nat Immunol. 2007;8:705-14.

52. Ranuncolo SM, Polo JM, Melnick A. BCL6 represses CHEK1 and suppresses DNA damage pathways in normal and malignant B-cells. Blood Cells Mol Dis. 2008;41:95-99.

53. Naugler WE, Sakurai T, Kim S, et al. Gender disparity in liver cancer due to sex differences in MyD88-dependent IL-6 production. Science. 2007;317:121-4. 